\title{
HIGHLIGHTS
}

BONE

\section{Comparing the structural effects of denosumab and alendronate}

Denosumab, a fully human antibody against receptor activator of NF- $\kappa \mathrm{B}$ ligand, and alendronate, a bisphosphonate, have different mechanisms of action in preventing structural decay of bone in patients with postmenopausal osteoporosis. Research using noninvasive, high-resolution imaging has now shown that these two antiresorptive therapies also have differing effects on bone microarchitecture and strength.

"As denosumab suppresses bone remodeling more rapidly and more greatly than alendronate, a treatment that is in common use, we were interested in determining if it may also slow structural decay more than alendronate," says Dr Ego Seeman, lead author of the paper in Journal of Bone and Mineral Research that reported the study results. The investigators employed high-resolution peripheral quantitative CT (HR-pQCT), which enables imaging of fine structural details such as cortical and trabecular architecture, as well as quantitative CT (QCT) to demonstrate treatment-specific changes in bone morphology.

Postmenopausal women aged 50-70 years with a T-score between -2.0 and -3.0 (determined by dual X-ray absorptometry) were eligible for the multicenter, double-blind, phase II pilot study. Participants were randomly assigned to receive treatment with subcutaneous denosumab 60 mg every 6 months $(n=83)$, oral alendronate $70 \mathrm{mg}$ weekly $(n=82)$, or placebo $(n=82)$; all patients also received daily calcium and vitamin $\mathrm{D}$ supplementation. HR-pQCT of the distal radius and distal tibia and QCT of the distal radius were performed at baseline and after 6 and 12 months of treatment.

In the placebo group, total, cortical and trabecular volumetric BMD (vBMD) were reduced at 12 months compared with baseline values, whereas treatment with alendronate prevented this loss of vBMD. Notably, denosumab increased vBMD, with changes in total and cortical vBMD that were higher than those observed with alendronate.

The results indicate that, while alendronate slows bone loss, denosumab partly restores cortical density. "The reason for this is being studied, but our provisional data suggest that denosumab reduces cortical porosity by allowing filling or partial filling of porosity already present and by preventing the appearance of newly excavated pores more so than does alendronate," says Dr Seeman.

Serum levels of the bone turnover markers CTX and P1NP decreased substantially with both treatments (to a greater extent with denosumab) but only slightly with placebo. In addition, density-weighted polar moment of inertia, which was used as an estimate of bone strength, increased following treatment with denosumab or alendronate, but more

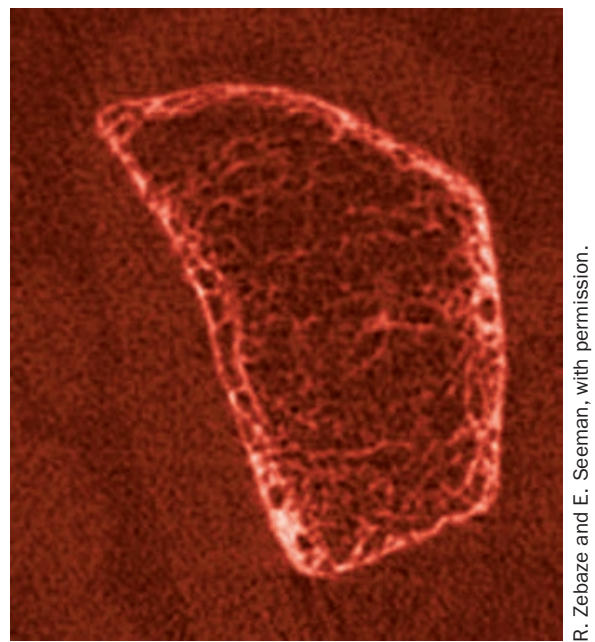

greatly with denosumab. Whether the greater effects of denosumab on cortical bone strength compared with alendronate will translate into reduced fracture risk at cortical sites remains to be studied.

"Porosity is an important cause of bone fragility," adds Dr Seeman. "The aim of treatment is to prevent the structural decay of bone or to attempt to reverse it before it becomes so severe that it is irreparable. Future studies will be focused on examining the effects of treatment on porosity measured by a new method of analysis and using this information to attempt to target treatment at persons at high risk of fracture."

\section{Sarah Price}

Original article Seeman, E. et al. Microarchitectural deterioration of cortical and trabecular bone: differing effects of denosumab and alendronate. J. Bone Miner. Res. doi:10.1002/jbmr.81 